\title{
Article \\ On the Periodic Solutions for the Perturbed Spatial Quantized Hill Problem
}

\author{
Elbaz I. Abouelmagd 1,*(D), Sawsan Alhowaity ${ }^{2} \mathbb{D}$, Zouhair Diab ${ }^{3} \mathbb{D}$, Juan L. G. Guirao ${ }^{4,5,6} \mathbf{D}^{\mathbb{D}}$ \\ and Mahmoud H. Shehata ${ }^{1}$ (D)
}

1 Celestial Mechanics and Space Dynamics Research Group (CMSDRG), Astronomy Department, National Research Institute of Astronomy and Geophysics (NRIAG), Helwan 11421, Cairo, Egypt; arlmahmoud925@gmail.com

2 Department of Mathematics, College of Science \& Humanities, Shaqra University, Shaqra 15551, Saudi Arabia; salhowaity@su.edu.sa

3 Department of Mathematics and Computer Science, Larbi Tebessi University, Tebessa 12002, Algeria; zouhair.diab@univ-tebessa.dz

4 Departamento de Matemáca Aplicada y Estadística, Universidad Politécnica de Cartagena, 30202 Cartagena, Spain; juan.garcia@upct.es

5 Department of Mathematics, Faculty of Science, King Abdulaziz University, P.O. Box 80203, Jeddah 21589, Saudi Arabia; jlgarcia@kau.edu.sa

6 Laboratory of Theoretical Cosmology, International Centre of Gravity and Cosmos, Tomsk State University of Control Systems and Radioelectronics (TUSUR), 634050 Tomsk, Russia

* Correspondence: elbaz.abouelmagd@nriag.sci.eg or eabouelmagd@gmail.com; Tel.: +20-1020976040

check for updates

Citation: Abouelmagd, E.I.; Alhowaity, S.; Diab, Z.; Guirao, J.L.G.; Shehata, M.H. On the Periodic Solutions for the Perturbed Spatial Quantized Hill Problem. Mathematics 2022, 10, 614. https://doi.org/ $10.3390 /$ math10040614

Academic Editors: Mikhail E.

Semenov and António M. Lopes

Received: 22 December 2021 Accepted: 14 February 2022 Published: 17 February 2022

Publisher's Note: MDPI stays neutral with regard to jurisdictional claims in published maps and institutional affiliations.

Copyright: (c) 2022 by the authors. Licensee MDPI, Basel, Switzerland. This article is an open access article distributed under the terms and conditions of the Creative Commons Attribution (CC BY) license (https:// creativecommons.org/licenses/by/ $4.0 /)$.
Abstract: In this work, we investigated the differences and similarities among some perturbation approaches, such as the classical perturbation theory, Poincaré-Lindstedt technique, multiple scales method, the KB averaging method, and averaging theory. The necessary conditions to construct the periodic solutions for the spatial quantized Hill problem-in this context, the periodic solutions emerging from the equilibrium points for the spatial Hill problem-were evaluated by using the averaging theory, under the perturbation effect of quantum corrections. This model can be used to develop a Lunar theory and the families of periodic orbits in the frame work for the spatial quantized Hill problem. Thereby, these applications serve to reinforce the obtained results on these periodic solutions and gain its own significance.

Keywords: quantized Hill problem; averaging theory; periodic solution

\section{Introduction}

A three-body problem plays a vital role in space science; in particular, the related fields of solar system motions, stars, planets, and their moons. The model of this problem can be used to characterize the dynamical behavior of the most stellar and planetary systems and to give accurate pictures on the motion. The three-body problem acquisition is important due to its application, not only in space science, but also in many fields, such as applied mathematics and mathematical physics. In addition, it is considered the simplest nonintegrable dynamical system in space dynamics, and it can be approximated to more simple systems, such as the perturbed two-body problem [1], Robe's restricted problem [2,3], and Hill's problem [4,5].

Periodic solutions of a dynamical system are solutions that characterize some repeated phenomena identically at regular intervals. These solutions play a vital role in many branches of science, such as physics and engineering, but appear in celestial mechanics, to study the dynamical structures of the two-body problem [6,7]; an analysis the infinitesimal body motion within the frame of the three-body problem [8,9] or N-body problem [10-12].

Considerable contributions have been made to the periodic solutions of either the unperturbed Kepler problem or the perturbed problem. For example, in [6], the authors 
have explored the existence of two periodic orbits at each energy level, in the framework of the anisotropic Kepler system, which emerges from elliptic orbits of the Kepler motion with high eccentricity, when the parameter of the anisotropy is small. Some interesting works have been constructed to analyze the periodic solutions [13-15].

The periodic solutions have particular significance in a three-body problem, regarding its extended applications in both space dynamics and celestial mechanics. Thereby, many dynamical systems that could be studied by considering the problem of the restricted three-body. Some of these systems have applications in space missions for spacecrafts in the planet-Moons systems (such as the Earth-Moon system). Further, this problem has application in stellar systems, to study the behavior of exoplanets in the proximity of one or both objects of a binary star system [16-18].

In fact, periodic solutions have considerable significance, because most of the natural phenomena in physical and engineering sciences, as well as celestial mechanics, could be characterized by periodic solutions of dynamical systems, characterized by ordinary or partial differential equations systems. These systems have a wide variety of applications, i.e., in physical, mathematical, and engineering sciences, as well as in the fields of biology, chemistry, and neural networks [19-22].

Many methods have been developed to analyze periodic solutions, such as the averaging method, Poincaré-Lindstedt technique, Krylov-Bogoliubov-Mitropolsky (KBM), and multiple scale methods, [23,24]. Periodic solutions are everywhere in the analysis of dynamical systems. Each field of science, in particularly, celestial mechanics, has its own oscillatory phenomena, which can be described by periodic solutions.

In this work, we aimed to find the periodic solutions of the dynamical system of the spatial quantized Hill problem. Thus, we evaluated the equilibria points of the linear system, and the necessary conditions were used to calculate the periodic solutions arising from the equilibria points for the spatial quantized Hill problem, by using the averaging theory. This system was constructed for the first time by Abouelmagd et al. (2020) [5]; this motivated us to study the dynamical structures of this system, through finding its own periodic solutions.

\section{Perturbation Techniques}

Perturbation techniques play substantial roles in analyzing non-linear dynamics; they have become a driving force, "pushing" mathematical researchers to extreme efforts, to explore and characterize the features of dynamical systems. These methods are considered excellent tools, and are designed to be applied in many fields.

In this chapter, we shed light on techniques that could be used to construct appropriate analytical periodic solutions of perturbed dynamical systems. Moreover, these techniques could be applied to some problems, where closed form solutions do not exist or where the exact solutions are either impossible or unrealistic of their physical meanings. In general, these systems are, often, neither linear nor autonomous in nature.

Now, we assume that $\mathcal{H}$ is an $n$-dimensional Hamiltonian system defined in terms of conjugate variables $(\mathbf{X}, \mathbf{Y})$, where $\mathbf{X} \in \Gamma, \Gamma$ is an open set of $\mathbb{R}^{n}$ and $\mathbf{Y} \in T^{n}$, here $T$ denotes the standard one-dimensional torus. Then, a nearly integrable Hamiltonian system $\mathcal{H}$ can be read as

$$
\mathcal{H}(\mathbf{X}, \mathbf{Y})=h(\mathbf{X}, \mathbf{Y})+\varepsilon f(\mathbf{X}, \mathbf{Y})
$$

where $h$ and $f$ are analytical functions called integrable (or unperturbed) Hamiltonian and the perturbing function, respectively. However, $\varepsilon$ is a small parameter, which measures the size of the perturbation force.

In the case of $\varepsilon=0$, the Hamiltonian function is given by

$$
\mathcal{H}(\mathbf{X}, \mathbf{Y})=h(\mathbf{X}, \mathbf{Y})
$$


using Hamiltonian relation (2), the associated equations of motion are given as

$$
\begin{aligned}
& \dot{\mathbf{X}}=0 \\
& \dot{\mathbf{Y}}=\mathbf{w}(\mathbf{X}),
\end{aligned}
$$

where, denoted with a dot, it is used for the derivatives, with respect to time, $\mathbf{w}$ is the frequency vector, defined as $\mathbf{w}=\partial h(\mathbf{X}) / \partial \mathbf{X}$.

The integration of System (3) is

$$
\begin{aligned}
& \mathbf{X}(t)=\mathbf{X}_{0} \\
& \mathbf{Y}=\mathbf{w}_{0} t+\mathbf{X}_{0},
\end{aligned}
$$

where $\mathbf{X}_{0}=\mathbf{X}(0)$ and $\mathbf{w}_{0}=\mathbf{w}(\mathbf{X}(0))$, Solution (4) shows that the variable $\mathbf{X}$ is constant, while its conjugate varies linearly with time.

In the case of the perturbing force, it has its own effect $(\varepsilon \neq 0)$, the Hamiltonian function is given in Relation (1); the associated equations of motion are

$$
\begin{aligned}
& \dot{\mathbf{X}}=-\varepsilon \frac{\partial f}{\partial \mathbf{Y}} \\
& \dot{\mathbf{Y}}=\mathbf{w}(\mathbf{X})+\varepsilon \frac{\partial f}{\partial \mathbf{X}} .
\end{aligned}
$$

System (5) may not be integrable and chaotic motion could appear.

\subsection{On Perturbation Techniques}

\subsubsection{Importance of Perturbation Techniques}

Physical phenomena in nature are mostly nonlinear and non-autonomous in their structures. The descriptions of these phenomena, within a linear sense, are not realistic, and present inaccurate information about their behaviour. Thus, it is necessary to preform the nonlinear dynamical systems, which describe these phenomena, but there are more difficulties in treating these systems by direct methods, and the perturbation techniques are considered the best choices in most cases. The perturbation techniques are employed for the dynamical systems, which consist of ordinary or partial differential equations.

Numerous methods can be used to obtain periodic solutions. For example the averaging method, for details, see [25-29]. The Liouville-Green method, which is known as the LG or WKB method, Lyapunov's theorem, Poincaré-Lindstedt technique, and KBM method [30,31]. There are more methods, such as the straightforward expansion technique (classical perturbation theory), but this method may fail in removing secular terms. Moreover, the multiple scales method is considered one of the strongest techniques in obtaining periodic solutions [32].

\subsubsection{Advantages and Disadvantages of Perturbation Techniques}

Exact solution of the dynamical system is rare, not only in celestial mechanics, but also in branches of applied mathematics: quantum mechanics, fluid mechanics, solid mechanics, and theoretical physics. This concerns nonlinearity behavior of a physical phenomenon. Therefore, engineers, physicists, and mathematicians are forced to find approximate solutions for mathematical models. These solutions may be purely analytical, purely numerical, or a combination of analytical and numerical techniques.

Perturbation techniques provide the most multilateral tools obtainable in non-linear sets of differential equations, which can be applied and employed to even more complex models. However, perturbation techniques have their own limitations, which mainly depend on presumptions, and very small parameters must appear in the prevailing equations. 


\subsection{Validity of Perturbation Techniques}

Many applications of perturbation methods are not available without the existence of a small parameter. An overwhelming number of non-linear dynamical systems, in particular, have "strong non-linearity" and no small parameters. In some estimation cases, this parameter is more the result of a technical procedure than scientific methodology. The convenient selection of small parameters could lead to intelligible results. On the contrary, incorrect choice for these parameters create inaccurate or even unrealistic solutions. Even if there exist appropriate small parameters that the perturbation methods provide us with by analytical solutions, they are adequate in cases of bounding the parameters.

The structures and analyses of perturbation approaches, such as classical perturbation theory, Poincaré-Lindstedt technique, multiple scales method, Krylov-Bogoliubov (KB) averaging method and averaging theory are familiar in the literature, as we have mentioned in the previous subsections. However, we will show the differences and similarities among the methods and rationales in choosing the averaging theory to find the periodic solution for the spatial quantized Hill problem.

The aforementioned techniques demand that the dynamical system be weakly nonlinear or weakly non-autonomous, meaning that those terms in the equation, including non-linearity or non-autonomy, are small. Alternatively systems of this structure can be thought of as almost linear or quasi-linear. One consequence of almost linear systems is that differential equations will have linear terms and small non-linear or non-autonomous terms separated from each other. These techniques can be applied to nearly integrable Hamiltonian systems, as in System (5).

The classical perturbation theory is called the straightforward expansion technique. It is used to find analytically solutions in the power series of the following form:

$$
\mathbf{X}(t, \varepsilon)=\sum_{k=0}^{\infty} \varepsilon^{k} \mathbf{X}_{k}(t) .
$$

In general, this method has secular terms that will provide unbounded solutions in the case of long intervals. Straightforward application of the classical perturbation theory to periodic nonlinear motion gives a result with secular terms, which will be proportional with time, in spite of the fact that the behavior of motion is known to be bounded. One of the common approaches used to remove those unwanted secular terms is the PoincaréLindstedt technique (also called the continuation method).

The multiple-scales technique is a generalized formula for the Poincaré-Lindstedt method. The latter method is dependant on the angular velocity of the non-linear oscillation and it may depend on its amplitude. The angular velocity is expanded in an asymptotic series, and the coefficients of each term in the series are evaluated in such a way that the solution has no secular terms.

In a similar manner, in the multiple-scales technique, the solution varies on fast and slow time separation scales. The first variable, "fast time scale", with respect to the first linear order in Poincaré-Lindstedt expansion, and every slow scale, matches with the second and higher terms in this expansion. The major difference is that multiple scales suppose that the coefficients of each scale are fixed, equal, and employ a variationof-parameters approach, considering the integration constants that appear in the linear solution be functions in the slow time scales variables. This process results in the dynamical system of partial differential equations at each order that has to be solved, to obtain a uniform solution of the dynamical system. The multiple-scales technique is a much more powerful tool than the Poincaré-Lindstedt method, because the former "admits" the constants compared to the slow time scale variables, while the latter only introduces one free variable at each order in the perturbed parameter. However, this method may result in a system, where the solution for obtaining periodicity conditions is more difficult than the main system. 
The Krylov-Bogolyubov averaging method is a technique used to find the periodic solution of a non-linear system, based on the averaging principle, where the exact system is replaced by an averaged one. To obtain the solution of the perturbed system or non-linear motion, using this method, the constants of linear motion vary slowly with time $t$ and the perturbation parameter $\varepsilon$. The significance of this method is that a general averaging approach is developed and proves that the solutions of the averaged systems give precise approximation to the original system [7].

Averaging is a mathematical method to replace a given field by its own average over a specified variable, such as time or an angular variable to get asymptotic approximation to the original system, aiming to obtain a periodic solution. In dynamical systems, the averaging method or the averaging theory utilizes systems, including time scale separation: fast oscillation versus a slow drift. We propose averaging over a certain interval of time to iron out the fast oscillation and monitor the qualitative behaviour from the resulting dynamics. It is a familiar problem where a trade-off exists between how perfect the approximate solution is balanced by how much time it holds to be similar to the original solution [27].

In this work, we used the averaging theory of dynamical systems, because it is especially worthy in the case of systems that can have isolated periodic orbits, such as in this case. In the next section, we will apply this method to Hill's version of the quantized three-body problem to find periodic solutions.

\section{Mathematical Model}

Hill's version of the quantized three-body problem was derived and analyzed for first time in [5], and the equations of motion are given by

$$
\begin{aligned}
\ddot{\xi}-2 \dot{\eta} & =3 \xi+2\left(\alpha_{1}-\alpha_{11}\right)-\frac{1}{r^{3}}\left[1+\frac{2 \alpha_{21}}{r}+\frac{3 \alpha_{22}}{r^{2}}\right] \xi, \\
\ddot{\eta}+2 \dot{\xi} & =-\frac{1}{r^{3}}\left[1+\frac{2 \alpha_{21}}{r}+\frac{3 \alpha_{22}}{r^{2}}\right] \eta, \\
\ddot{\zeta} & =-\zeta-\frac{1}{r^{3}}\left[1+\frac{2 \alpha_{21}}{r}+\frac{3 \alpha_{22}}{r^{2}}\right] \zeta .
\end{aligned}
$$

System (7) characterizes the perturbed spatial Hill problem, the system is perturbed by quantum corrections; hence, this system is called the spatial quantized Hill problem (SQHP). Furthermore, this system is considered a "limiting case" from the spatial quantized restricted three-bodies problem; it was developed in [33]. In addition, this system can be described by the style of writing of the restricted three-body problem,

$$
\begin{aligned}
\ddot{\xi}-2 \dot{\eta} & =\Psi_{\xi}(\xi, \eta, \zeta), \\
\ddot{\eta}+2 \dot{\xi} & =\Psi_{\eta}(\xi, \eta, \zeta), \\
\ddot{\zeta} & =\Psi_{\zeta}(\xi, \eta, \zeta),
\end{aligned}
$$

where

$$
\Psi(\xi, \eta, \zeta)=\frac{1}{2}\left[3 \xi^{2}+4\left(\alpha_{1}-\alpha_{11}\right) \xi-\zeta^{2}\right]+\frac{1}{r}\left[1+\frac{\alpha_{21}}{r}+\frac{\alpha_{22}}{r^{2}}\right],
$$

and $\Psi_{\xi}, \Psi_{\beta}, \Psi_{\zeta}$ refer to the partial derivatives of the potential function, with respect to the variables $\xi, \beta$, and $\xi$, while the separation distance $r$ is given by $r=\sqrt{\xi^{2}+\beta^{2}+\zeta^{2}}$.

Here, $\alpha_{1}, \alpha_{11}$, and $\alpha_{21}$ are very small quantities with order $\mathcal{O}\left(1 / c^{2}\right)$, while $\alpha_{22}$ is more smaller with order $\mathcal{O}\left(1 / c^{3}\right)$, where $c$ is the speed of light; for comprehensive details, 
see [33]. Since $\alpha_{1}, \alpha_{11}$, and $\alpha_{21}$ have the same order, then $\alpha_{1}-\alpha_{11} \cong 0$, and System (8) and the potential function can be simplified and rewritten

$$
\begin{aligned}
\ddot{\xi}-2 \dot{\eta} & =\bar{\Psi}_{\xi}(\xi, \eta, \zeta), \\
\ddot{\eta}+2 \dot{\zeta} & =\bar{\Psi}_{\eta}(\xi, \eta, \zeta), \\
\ddot{\zeta} & =\bar{\Psi}_{\zeta}(\xi, \eta, \zeta),
\end{aligned}
$$

where

$$
\Psi(\xi, \eta, \zeta)=\frac{1}{2}\left[3 \xi^{2}-\zeta^{2}\right]+\frac{1}{r}\left[1+\frac{\alpha_{21}}{r}+\frac{\alpha_{22}}{r^{2}}\right] .
$$

Although many different analyses in celestial mechanics have been accomplished in the framework of the three-body problem, but there are also many dynamical concepts can be carried out within frame of Hill problem [34] without loosing the required accuracy underlying of using simple model. Furthermore, the perturbed model of this problem can be used to study the effect of some perturbed forces on the dynamical properties, such as the emerging periodic solutions from the equilibria points, which will be analyzed in the next sections.

\section{Periodic Solutions}

Periodic solutions or periodic orbits are considered one the major reasons of stability and are continuous in life; for example, the periodicity motion of the Sun and Moon. When the Hill model was constructed, the researchers devoted their work toward calculating the families of periodic orbits. More work was developed to analyze the lunar theory dependent on Hill's problem. For the importance of Hill's problem, we to explore the presence of periodic solutions emerged from equilibria points by underlying SQHP.

By taking $\alpha_{1}=\alpha_{11}=\alpha_{21}=1.5 \times 10^{-3}, \alpha_{22}=1.5 \times 10^{-5}$ of the differential System (7), we get the following equilibrium points $E_{1}=(\delta, 0,0)$ and $E_{2}=(-\delta, 0,0)$ where $\delta \approx 0.694035$.

Thus, we study the presence of periodic solutions, which emerge from equilibria points $E_{1}$ and the same ones are valid for $E_{2}$. To study the motion around or in the proximity of the equilibria points $E_{1}$ and $E_{2}$, we first have to linearize System (8). Thus, we impose that $\xi=x_{1}-\delta, \eta=y_{1}, \zeta=z_{1}$, where $x_{1}, y_{1} z_{1}$ are very small displacements from the equilibria points; thereby, the associated linear system to non-linear System (8) are given by

$$
\begin{aligned}
\frac{d^{2} x_{1}}{d t^{2}}-2 \frac{d y_{1}}{d t}-\alpha x_{1} & =0, \\
\frac{d^{2} y_{1}}{d t^{2}}+2 \frac{d x_{1}}{d t}+\beta y_{1} & =0, \\
\frac{d^{2} z_{1}}{d t^{2}}+\gamma z_{1} & =0,
\end{aligned}
$$

with $\alpha \approx 9.03, \beta \approx 3$ and $\gamma \approx 3$.

Since the means of the averaging theory is one of the powerful tools for finding periodic solutions, then we will apply this method to study the existence of periodic solutions of the following system

$$
\begin{aligned}
\frac{d^{2} x_{1}}{d t^{2}}-2 \frac{d y_{1}}{d t}-\alpha x_{1} & =\varepsilon \mathcal{F}_{1}\left(t, x_{1}, \frac{d x_{1}}{d t}, y_{1}, \frac{d y_{1}}{d t},, z_{1}, \frac{d z_{1}}{d t}\right), \\
\frac{d^{2} y_{1}}{d t^{2}}+2 \frac{d x_{1}}{d t}+\beta y_{1} & =\varepsilon \mathcal{F}_{2}\left(t, x_{1}, \frac{d x_{1}}{d t}, y_{1}, \frac{d y_{1}}{d t}, z_{1}, \frac{d z_{1}}{d t}\right) \\
\frac{d^{2} z_{1}}{d t^{2}}+\gamma z_{1} & =\varepsilon \mathcal{F}_{3}\left(t, x_{1}, \frac{d x_{1}}{d t}, y_{1}, \frac{d y_{1}}{d t}, z_{1}, \frac{d z_{1}}{d t}\right)
\end{aligned}
$$


where $\varepsilon$ is the perturbation parameter; it is a very small quantity and the functions $\mathcal{F}_{1}, \mathcal{F}_{2}$, $\mathcal{F}_{3}$ represent the non-linear terms, which will be ignored when $\varepsilon=0$, but this function satisfies the following properties:

- $\mathcal{F}_{1}, \mathcal{F}_{2}, \mathcal{F}_{3}$ are smooth functions;

- $\mathcal{F}_{1}, \mathcal{F}_{2}, \mathcal{F}_{3}$ are periodic functions in variable $t$;

- $\mathcal{F}_{1}, \mathcal{F}_{2}, \mathcal{F}_{3}$ are resonance in $\iota_{1}: \iota_{2}$ with periodic solutions for System (12); where $\iota_{1}$ and $\iota_{2}$ are primes numbers.

There is a unique singular point for the unperturbed System (12) at the origin with eigenvalues $\pm \Omega, \pm \omega_{1} i, \pm \omega_{2} i$, where $\Omega \approx 2.51, \omega_{1} \approx 2.07$ and $\omega_{2} \approx 1.73$. In the phase space

$$
\left(x_{1}, \frac{d x_{1}}{d t}, y_{1}, \frac{d y_{1}}{d t}, z_{1}, \frac{d z_{1}}{d t}\right)
$$

the aforementioned system (the unperturbed system) has two planes filed of periodic solutions with the exception of the origin, where the periods of solutions are

$$
T_{1}=2 \pi / \omega_{1} \text { or } 2 \pi / \omega_{2},
$$

here, periods $T_{1}$ and $T_{2}$ are related to the eigenvalues $\pm \omega_{1} i$ or $\pm \omega_{2} i$, respectively. We will explore which of the periodic solutions continue for the perturbed System (13), where the parameter of perturbation $\varepsilon$ is small enough and there are two periods, either $\iota_{1} T_{1} / l_{2}$ or $\iota_{1} T_{2} / \iota_{2}$, for the perturbed function $\mathcal{F}_{i}$ for $i \in\{1,2,3\}$.

Consider $Z^{0}=\left(Z_{1}^{0}, Z_{2}^{0}\right)$, and $\mathcal{H}=\left(\mathcal{H}_{1}, \mathcal{H}_{2}\right)$ for the System (13), with

$$
\begin{aligned}
\mathcal{H}_{1}\left(Z^{0}\right) & =\frac{1}{\iota_{1} T_{2}} \int_{0}^{\iota_{1} T_{2}}\left\langle\left(\cos \left(\omega_{2} t\right),-\sin \left(\omega_{2} t\right)\right),\left(\mathcal{F}_{5}^{*}(t), \mathcal{F}_{6}^{*}(t)\right)\right\rangle d t \\
& =\frac{1}{\iota_{1} T_{2}} \int_{0}^{\iota_{1} T_{2}}-\sin \left(\omega_{2} t\right) \mathcal{F}_{6}^{*}(t) d t \\
\mathcal{H}_{2}\left(Z^{0}\right) & =\frac{1}{p T_{2}} \int_{0}^{p T_{2}}\left\langle\left(\sin \left(\omega_{2} t\right), \cos \left(\omega_{2} t\right)\right),\left(\mathcal{F}_{5}^{*}(t), \mathcal{F}_{6}^{*}(t)\right)\right\rangle d t \\
& =\frac{1}{p T_{2}} \int_{0}^{p T_{2}} \cos \left(\omega_{2} t\right) \mathcal{F}_{6}^{*}(t) d t
\end{aligned}
$$

where $\langle$,$\rangle is the scalar product and \mathcal{F}_{5}^{*}(t)=0, \mathcal{F}_{6}^{*}=1.12 \mathcal{F}_{3}, \mathcal{F}_{3}=\mathcal{F}_{3}\left(\eta_{1}^{2}(t), \ldots, \eta_{6}^{2}(t)\right)$ and $\eta_{j}^{2}(t)=0, j=1, \ldots, 4$, while

$$
\begin{aligned}
& \eta_{5}^{2}(t)=0.50\left(Z_{1}^{0} \cos \left(\omega_{2} t\right)+Z_{2}^{0} \sin \left(\omega_{2} t\right)\right), \\
& \eta_{6}^{2}(t)=0.86\left(Z_{2}^{0} \cos \left(\omega_{2} t\right)-Z_{1}^{0} \sin \left(\omega_{2} t\right)\right) .
\end{aligned}
$$
where

Now, we impose that $Z^{0 *}=\left(Z_{1}^{0 *}, Z_{2}^{0 *}\right)$ is the zero of non-linear system $\mathcal{H}\left(Z^{0}\right)=0$

$$
\left|\frac{\partial \mathcal{H}}{\partial Z^{0}}\right| \neq 0 \text { when } Z^{0}=Z^{0 *},
$$

Then, we can state that the system has a simple zero [35]. The expression of simple zero or pole is used to describe the zero or pole of order one, and sometimes the term of "degree" is used instead of "order". The property of this zero or pole leading to this zero can be isolated and its neighbourhood has no other zero.

We emphasize that if the Malkin bifurcation function $\mathcal{H}$ has a simple zero $Z^{0 *}$ and the solution of the unperturbed system has a period $T_{2}$ by using initial value $Z^{0 *}$, then the perturbed system will has also $T_{2}-$ periodic solution. 
The periodic solution of the dynamical System (13) is considered the main result in this work, where this solution will bifurcate from the $T_{2}$-periodic solution of the unperturbed system; hence, we present the following theorem:

Theorem 1. We impose that $\iota_{1}$ and $\iota_{2}$ are prime numbers, and

- $\quad \mathcal{F}_{1}, \mathcal{F}_{2}$, and $\mathcal{F}_{3}$ are smooth functions of System (13)

- $\quad \mathcal{F}_{1}, \mathcal{F}_{2}$, and $\mathcal{F}_{3}$ are periodic, with period $\iota_{1} T_{2} / \iota_{2}$ in variable $t$

For each simple zero, $Z^{0 *} \neq 0$ of the non-linear system $\mathcal{H}\left(Z^{0}\right)=0$ when $\varepsilon \neq 0$ and enough small, we can find a periodic solution for the perturbed System (13) takes the form $\left(x_{1}(t, \varepsilon), y_{1}(t, \varepsilon)\right.$, $\left.z_{1}(t, \varepsilon)\right)$ and tends to the periodic solution

$\left(x_{1}(t), y_{1}(t), z_{1}(t)\right)=\left.\left(\eta_{1}^{2}(t), \eta_{3}^{2}(t), \eta_{5}^{2}(t)\right)\right|_{Z^{0} Z^{0 *}}$ of the unperturbed System (12) traveled $\iota_{1}$ times.

We will presented the proof of Theorem 1 in Section 5 . Further, the following corollary is considered the application of this theorem and its proof will be presented in Section 6 .

Corollary 1. Considering that $\mathcal{F}_{1}\left(t, x_{1}, \dot{x}_{1}, y_{1}, \dot{y}_{1}, z_{1}, \dot{z}_{1}\right)=0, \mathcal{F}_{2}\left(t, x_{1}, \dot{x}_{1}, y_{1}, \dot{y}_{1}, z_{1}, \dot{z}_{1}\right)=0$, $\mathcal{F}_{3}\left(t, x_{1}, \dot{x}_{1}, y_{1}, \dot{y}_{1}, z_{1}, \dot{z}_{1}\right)=z^{5}+\sin \left(\omega_{2} t\right) \dot{z}^{2}$. Thus, the System (13) with $\varepsilon \neq 0$ is enough small, and has one periodic solution $\left(x_{1}(t, \varepsilon), y_{1}(t, \varepsilon), z_{1}(t, \varepsilon)\right)$ approximating to the periodic solutions $\left(x_{1}(t), y_{1}(t), z_{1}(t)\right)=\left.\left(\eta_{1}^{2}(t), \eta_{3}^{2}(t), \eta_{5}^{2}(t)\right)\right|_{Z^{0}=Z^{0 *}}$ of $(2)$ when $\varepsilon \rightarrow 0$, given by $Z^{0 *}=$ $(0,-2.11)$.

Now, we impose that $Y^{0}=\left(Y_{1}^{0}, Y_{2}^{0}\right)$, and considering the Malkin bifurcation function $\overline{\mathcal{H}}=\left(\mathcal{H}_{3}, \mathcal{H}_{4}\right)$ for the System (13) controlled by

$$
\begin{aligned}
\mathcal{H}_{3}\left(Y^{0}\right) & =\frac{1}{p T_{1}} \int_{0}^{p T_{1}}\left\langle\left(\cos \left(\omega_{1} t\right),-\sin \left(\omega_{1} t\right)\right),\left(\mathcal{F}_{3}^{*}(t), \mathcal{F}_{4}^{*}(t)\right)\right\rangle d t \\
& =\frac{1}{p T_{1}} \int_{0}^{p T_{1}}\left(\cos \left(\omega_{1} t\right) \mathcal{F}_{3}^{*}(t)-\sin \left(\omega_{1} t\right) \mathcal{F}_{4}^{*}(t)\right) d t, \\
\mathcal{H}_{4}\left(Y^{0}\right) & =\frac{1}{p T_{1}} \int_{0}^{p T_{1}}\left\langle\left(\sin \left(\omega_{1} t\right), \cos \left(\omega_{1} t\right)\right),\left(\mathcal{F}_{3}^{*}(t), \mathcal{F}_{4}^{*}(t)\right)\right\rangle d t \\
& =\frac{1}{p T_{1}} \int_{0}^{p T_{1}}\left(\cos \left(\omega_{1} t\right) \mathcal{F}_{4}^{*}(t)+\sin \left(\omega_{1} t\right) \mathcal{F}_{3}^{*}(t)\right) d t,
\end{aligned}
$$

where

$$
\mathcal{F}_{3}^{*}=-0.45 \mathcal{F}_{1}, \quad \mathcal{F}_{4}^{*}=-1.46 \mathcal{F}_{2}
$$

with $\mathcal{F}_{i}=\mathcal{F}_{i}\left(\eta_{1}^{1}(t), \ldots, \eta_{6}^{1}(t)\right), i \in\{1,2\}$ and $\eta_{j}^{2}(t)=0, j=5,6$,

$$
\begin{aligned}
& \eta_{1}^{1}(t)=0.12\left(Y_{2}^{0} \cos \left(\omega_{1} t\right)-Y_{1}^{0} \sin \left(\omega_{1} t\right)\right), \\
& \eta_{2}^{1}(t)=-0.26\left(Y_{1}^{0} \cos \left(\omega_{1} t\right)+Y_{2}^{0} \sin \left(\omega_{1} t\right)\right), \\
& \eta_{3}^{1}(t)=-0.41\left(Y_{1}^{0} \cos \left(\omega_{1} t\right)+Y_{2}^{0} \sin \left(\omega_{1} t\right)\right), \\
& \eta_{4}^{1}(t)=-0.85\left(Y_{2}^{0} \cos \left(\omega_{1} t\right)-Y_{1}^{0} \sin \left(\omega_{1} t\right)\right),
\end{aligned}
$$

As we aforementioned with the existence of a simple zero $Y^{0 *}$ of the Malkin bifurcation function $\overline{\mathcal{H}}$, one will obtain from $T_{1}$-periodic solution of the unperturbed system, with initial value $Y^{0 *}$, emerges the solution of a perturbed system with $T_{1}$-periodic solution, because the simple zero can be isolated and its neighbourhood has no other zero.

The second result related to the periodic solutions, which is associated to unperturbed System (13), will be stated in the following theorem: 
Theorem 2. We impose that $\iota_{1}$ and $\iota_{2}$ are primes numbers and

- $\quad \mathcal{F}_{1}, \mathcal{F}_{2}$, and $\mathcal{F}_{3}$ are smooth functions of System (13)

- $\mathcal{F}_{1}, \mathcal{F}_{2}$, and $\mathcal{F}_{3}$ are periodic with period $\iota_{1} T_{1} / \iota_{2}$ in variable $t$

For each simple zero $\mathrm{Y}^{0 *} \neq 0$ of the non-linear system $\overline{\mathcal{H}}\left(Y^{0}\right)=0$, when $\varepsilon \neq 0$ is enough small, then the perturbed System (13) has a periodic solution $\left(x_{1}(t, \varepsilon), y_{1}(t, \varepsilon), z_{1}(t, \varepsilon)\right)$ going to the periodic solution $\left(x_{1}(t), y_{1}(t), z_{1}(t)\right)=\left.\left(\eta_{1}^{1}(t), \eta_{3}^{1}(t), \eta_{5}^{1}(t)\right)\right|_{\gamma^{0}=\gamma^{0 *}}$ of the unperturbed System (12) traveled $\iota_{1}$ times.

The application of Theorem 2 can be stated in the following corollary:

Corollary 2. Considering that $\mathcal{F}_{1}\left(t, x_{1}, \dot{x}_{1}, y_{1}, \dot{y}_{1}, z_{1}, \dot{z}_{1}\right)=\sin \left(\omega_{1} t\right)+x_{1}+3 x_{1}^{2} y_{1}+y_{1}$, $\mathcal{F}_{2}\left(t, x_{1}, \dot{x}_{1}, y_{1}, \dot{y}_{1}, z_{1}, \dot{z}_{1}\right)=\cos \left(\omega_{1} t\right)-\left(\dot{y}_{1}\right)^{2}+x_{1}, \mathcal{F}_{3}\left(t, x_{1}, \dot{x}_{1}, y_{1}, \dot{y}_{1}, z_{1}, \dot{z}_{1}\right)=0$. Then the System (13) for $\varepsilon \neq 0$ sufficiently small has one periodic solution $\left(x_{1}(t, \varepsilon), y_{1}(t, \varepsilon), z_{1}(t, \varepsilon)\right)$ tending to the periodic solutions $\left(x_{1}(t), y_{1}(t), z_{1}(t)\right)=\left.\left(\eta_{1}^{1}(t), \eta_{3}^{1}(t), \eta_{5}^{1}(t)\right)\right|_{\gamma^{0}=\gamma^{0 *}}$ of System (12) when $\varepsilon \rightarrow 0$, given by $Y^{0 *}=(2.59,9.21)$.

The proof of Corollary 2 will be given in Section 6.

\section{Proof of the Theorems 1 and 2}

To accomplish the proofs of Theorems 1 and 2, we will use the following variables:

$$
\left(x_{1}, x_{2}, y_{1}, y_{2}, z_{1}, z_{2}\right)=\left(x_{1}, \frac{d x_{1}}{d t}, y_{1}, \frac{d y_{1}}{d t}, z_{1}, \frac{d z_{1}}{d t}\right)
$$

Thus, the dynamical System (13) can be rewritten in the form of a first order in $\mathbb{R}^{6}$

$$
\begin{aligned}
& \frac{d x_{1}}{d t}=x_{2}, \quad \frac{d x_{2}}{d t}=\alpha x_{1}+2 y_{2}+\varepsilon \mathcal{F}_{1}\left(x_{1}, x_{2}, y_{1}, y_{2}, z_{1}, z_{2}\right), \\
& \frac{d y_{1}}{d t}=y_{2}, \quad \frac{d y_{2}}{d t}=-2 x_{2}-\beta y_{1}+\varepsilon \mathcal{F}_{2}\left(x_{1}, x_{2}, y_{1}, y_{2}, z_{1}, z_{2}\right) \text {, } \\
& \frac{d z_{1}}{d t}=z_{2}, \quad \frac{d z_{2}}{d t}=-\gamma z_{1}+\varepsilon \mathcal{F}_{3}\left(x_{1}, x_{2}, y_{1}, y_{2}, z_{1}, z_{2}\right) .
\end{aligned}
$$

It is clear that the perturbed System $(15)(\varepsilon \neq 0)$ can be reduced to the unperturbed System (12) when $\varepsilon=0$. Now we write the perturbed System (15) with the style that the linear part at the origin point will take the real Jordan expression, after that, we can change the variables to the following form:

$$
\left(x_{1}, x_{2}, y_{1}, y_{2}, z_{1}, z_{2}\right) \rightarrow\left(X_{1}, X_{2}, Y_{1}, Y_{2}, Z_{1}, Z_{2}\right)
$$

given by

$$
\left(\begin{array}{l}
x_{1} \\
x_{2} \\
y_{1} \\
y_{2} \\
z_{1} \\
z_{2}
\end{array}\right)=\left(\begin{array}{cccccc}
-0.32 & 0.32 & 0.0 & 0.12 & 0.0 & 0.0 \\
-0.81 & -0.81 & -0.26 & 0 & 0.0 & 0.0 \\
0.17 & 0.17 & -0.41 & 0 & 0.0 & 0.0 \\
0.44 & -0.44 & 0.0 & -0.85 & 0.0 & 0.0 \\
0.0 & 0.0 & 0.0 & 0.0 & 0.5 & 0.0 \\
0.0 & 0.0 & 0.0 & 0.0 & 0.0 & 0.86
\end{array}\right)\left(\begin{array}{c}
X_{1} \\
X_{2} \\
Y_{1} \\
Y_{2} \\
Z_{1} \\
Z_{2}
\end{array}\right)
$$


and

$$
\left(\begin{array}{l}
X_{1} \\
X_{2} \\
Y_{1} \\
Y_{2} \\
Z_{1} \\
Z_{2}
\end{array}\right)=\left(\begin{array}{cccccc}
-1.92 & -0.53 & 0.34 & -0.28 & 0.0 & 0.0 \\
1.92 & -0.53 & 0.34 & 0.28 & 0.0 & 0.0 \\
0.0 & -0.45 & -2.11 & 0.0 & 0.0 & 0.0 \\
-1.98 & 0.0 & 0.0 & -1.46 & 0.0 & 0.0 \\
0.0 & 0.0 & 0.0 & 0.0 & 2.0 & 0.0 \\
0.0 & 0.0 & 0.0 & 0.0 & 0.0 & 1.15
\end{array}\right)\left(\begin{array}{l}
x_{1} \\
x_{2} \\
y_{1} \\
y_{2} \\
z_{1} \\
z_{2}
\end{array}\right),
$$

the differential System (15) becomes

$$
\begin{aligned}
& \dot{X}_{1}=\Omega X_{1}+\varepsilon \mathcal{F}_{1}^{*}, \\
& \dot{X}_{2}=-\Omega X_{2}+\varepsilon \mathcal{F}_{2}^{*}, \\
& \dot{Y}_{1}=\omega_{1} Y_{2}+\varepsilon \mathcal{F}_{3}^{*}, \\
& \dot{Y}_{2}=-\omega_{1} Y_{1}+\varepsilon \mathcal{F}_{4}^{*}, \\
& \dot{Z}_{1}=\omega_{2} Z_{2}+\varepsilon \mathcal{F}_{5}^{*}, \\
& \dot{Z}_{2}=-\omega_{2} Z_{1}+\varepsilon \mathcal{F}_{6}^{*},
\end{aligned}
$$

where

$$
\begin{aligned}
& \mathcal{F}_{1}^{*}=-0.53 \mathcal{F}_{1}-0.28 \mathcal{F}_{2}, \\
& \mathcal{F}_{2}^{*}=-0.53 \mathcal{F}_{1}+0.28 \mathcal{F}_{2}, \\
& \mathcal{F}_{3}^{*}=-0.45 \mathcal{F}_{1}, \\
& \mathcal{F}_{4}^{*}=-1.46 \mathcal{F}_{2}, \\
& \mathcal{F}_{5}^{*}=0, \\
& \mathcal{F}_{6}^{*}=1.15 \mathcal{F}_{3},
\end{aligned}
$$

with $\mathcal{F}_{i}=\mathcal{F}_{i}\left(\eta_{1}, \ldots, \eta_{6}\right), i \in\{1,2,3\}$ and

$$
\begin{aligned}
& \eta_{1}=-0.32 X_{1}+0.32 X_{2}+0.12 Y_{2}, \\
& \eta_{2}=-0.81 X_{1}-0.81 X_{2}-0.26 Y_{1}, \\
& \eta_{3}=0.17 X_{1}+0.17 X_{2}-0.41 Y_{1}, \\
& \eta_{4}=0.44 X_{1}-0.44 X_{2}-0.85 Y_{2}, \\
& \eta_{5}=0.5 Z_{1}, \\
& \eta_{6}=0.86 Z_{2},
\end{aligned}
$$

To prove Theorems 1 and 2, we first depict the periodic solution of the unperturbed system through the following Lemma

Lemma 1. The periodic solutions $\left(X_{1}(t), X_{2}(t), Y_{1}(t), Y_{2}(t), Z_{1}(t), Z_{2}(t)\right)$ of System (17) when $\varepsilon=0$ are

$$
\left(0,0, Y_{1}^{0} \cos \left(\omega_{1} t\right)+Y_{2}^{0} \sin \left(\omega_{1} t\right), Y_{2}^{0} \cos \left(\omega_{1} t\right)-Y_{1}^{0} \sin \left(\omega_{1} t\right), 0,0\right),
$$

where $T_{1}$ is the period of motion, and

$$
\left(0,0,0,0, Z_{1}^{0} \cos \left(\omega_{2} t\right)+Z_{2}^{0} \sin \left(\omega_{2} t\right), Z_{2}^{0} \cos \left(\omega_{2} t\right)-Z_{1}^{0} \sin \left(\omega_{2} t\right)\right),
$$

here, $T_{2}$ is the period of motion.

Proof of Lemma 1. Since System (17) is linear when $\varepsilon=0$ thereby, the proof can be easily established. 
Proof of Theorem 1. We impose that $\mathcal{F}_{1}, \mathcal{F}_{2}$, and $\mathcal{F}_{3}$ of (13) are periodic functions in $t$ with period $\iota_{1} T_{1} / \iota_{2}$ where $\iota_{1}$ and $\iota_{2}$ are primes numbers. Hence, the same periodicity features are the same for System (17) and the periodic Solutions (19) with period $\iota_{1} T_{2}$. By applying Theorem A1 in [35] and using the same notation and terminology to the System (17), then we can write the System (17) in the following form

$$
\dot{X}(t)=H_{0}(t, X)+\varepsilon H_{1}(t, X)+\varepsilon^{2} H_{2}(t, X, \varepsilon)
$$


Then, we can consider

$$
X=\left(\begin{array}{c}
X_{1} \\
X_{2} \\
Y_{1} \\
Y_{2} \\
Z_{1} \\
Z_{2}
\end{array}\right), \quad H_{0}(t, X)=\left(\begin{array}{c}
\Omega X_{1} \\
-\Omega X_{2} \\
\omega_{1} Y_{2} \\
-\omega_{1} Y_{1} \\
\omega_{2} Z_{2} \\
-\omega_{2} Z_{1}
\end{array}\right), \quad H_{1}(t, X)=\left(\begin{array}{c}
\mathcal{F}_{1}^{*} \\
\mathcal{F}_{2}^{*} \\
\mathcal{F}_{3}^{*} \\
\mathcal{F}_{4}^{*} \\
\mathcal{F}_{5}^{*} \\
\mathcal{F}_{6}^{*}
\end{array}\right), \quad H_{2}(t, X, \varepsilon)=\left(\begin{array}{l}
0 \\
0 \\
0 \\
0 \\
0 \\
0
\end{array}\right) .
$$

In this context, the periodic solution of the unperturbed System $(17)(\varepsilon=0)$ will be studied within the Type (19) to continue as a periodic solution for the perturbed system (when $\varepsilon \neq 0$ is enough small). First, we characterize the different parameters, which are stated in Theorem A1 (see the Appendix in [35] for details) due to the certain case of the System (17). Now, we assume that $\rho_{1}>0$ and $\rho_{2}>0$, where $\rho_{1}>0$ is chosen to be small, while $\rho_{2}>0$ is chosen to be large. We also assume that $V$ is bounded and open subset of the plane $X_{1}=X_{2}=Y_{1}=Y_{2}=0$ of the form

$$
V=\left\{\left(0,0,0,0, Z_{1}^{0}, Z_{2}^{0}\right) \in \mathbb{R}^{6}: \rho_{1}<\sqrt{\left(Z_{1}^{0}\right)^{2}+\left(Z_{2}^{0}\right)^{2}}<\rho_{2}\right\} .
$$

Since $V$ is bounded and open subset of $\mathbb{R}^{2}$, we can choose two numbers $\rho_{1}>0, \rho_{2}>0$, such that

$$
V=\left\{(\beta(\alpha), \alpha) \in \mathbb{R}^{2}: \rho_{1}<\sqrt{\left(Z_{1}^{0}\right)^{2}+\left(Z_{2}^{0}\right)^{2}}<\rho_{2}\right\} .
$$

where $\alpha \in R^{2}$ and $\beta(\alpha) \in R^{4}$.

Now, we assume that $\alpha=Z^{0}=\left(Z_{1}^{0}, Z_{2}^{0}\right)$, then we characterize $V$ with the set $\left\{\alpha \in \mathbb{R}^{2}: \rho_{1}<\|\alpha\|<\rho_{2}\right\}$, being $\|\cdot\|$ the Euclidean norm in $\mathbb{R}^{2}$, while the function $\beta(\alpha)$ is defined as $\beta: C 1(V) \rightarrow \mathbb{R}^{4}$ such that $\beta(\alpha)=(0,0,0,0)$, here $C 1(V)$ refers to the closure of $V$. Hence, for the proposed system, one obtains

$$
\begin{aligned}
\mathcal{Z} & =\left\{\mathbf{z}_{\alpha}=(\beta(\alpha), \alpha), \alpha \in C 1(V)\right\} \\
& =\left\{\left(0,0,0,0, Z_{1}^{0}, Z_{2}^{0}\right) \in \mathbb{R}^{6}: \rho_{1} \leq \sqrt{\left(Z_{1}^{0}\right)^{2}+\left(Z_{2}^{0}\right)^{2}} \leq \rho_{2}\right\} .
\end{aligned}
$$

We take for each $\mathbf{z}_{\alpha} \in \mathcal{Z}$ the periodic solution

$$
\mathbf{x}\left(t, \mathbf{z}_{\alpha}\right)=\left(0,0,0,0, Z_{1}(t), Z_{2}(t)\right),
$$

controlled by System (19) of period $\iota_{1} T_{2}$. Calculating the matrix $M_{\mathbf{z}_{\alpha}}(t)$ of the linear System (17), which is called the fundamental matrix where $\varepsilon=0$ related to the $\iota_{1} T_{2}$ periodic solution $\mathbf{z}_{\alpha}=\left(0,0,0,0, Z_{1}^{0}, Z_{2}^{0}\right)$ and also $M_{\mathbf{z}_{\alpha}}(0)$ is the identity element in space $\mathbb{R}^{6}$; thus, one obtains

$$
M_{\mathbf{z}_{\alpha}}(t)=M(t)=\left(\begin{array}{cccccc}
e^{\Omega t} & 0 & 0 & 0 & 0 & 0 \\
0 & -e^{-\Omega t} & 0 & 0 & 0 & 0 \\
0 & 0 & \cos \left(\omega_{1} t\right) & \sin \left(\omega_{1} t\right) & 0 & 0 \\
0 & 0 & -\sin \left(\omega_{1} t\right) & \cos \left(\omega_{1} t\right) & 0 & 0 \\
0 & 0 & 0 & 0 & \cos \left(\omega_{2} t\right) & \sin \left(\omega_{2} t\right) \\
0 & 0 & 0 & 0 & -\sin \left(\omega_{2} t\right) & \cos \left(\omega_{2} t\right)
\end{array}\right) .
$$

There is no correlation between the matrix $M_{\mathbf{z}_{\alpha}}(t)$ and particular periodic solution $\mathbf{x}\left(t, \mathbf{z}_{\alpha}, 0\right)$. 
Now, we impose that the matrix $\hbar$ is defined by

$$
\hbar=M^{-1}(0)-M^{-1}\left(\iota_{1} T_{2}\right)
$$

then the matrix $\hbar$ is satisfied the stated assumptions (ii) in Theorem A1 (see the Appendix in [35] for details), where the matrix $\hbar$ and its determinant are given by

$$
\begin{gathered}
\hbar=\left(\begin{array}{cccccc}
1-e^{-2 \pi \Omega \iota_{1} / \omega_{2}} & 0 & 0 & 0 & 0 & 0 \\
0 & 1-e^{2 \pi \Omega \iota_{1} / \omega_{2}} & 0 & 0 & 0 & 0 \\
0 & 0 & 2 \sin ^{2}\left(\pi \iota_{1} \omega_{1} / \omega_{2}\right) & \sin \left(2 \pi \iota_{1} \omega_{1} / \omega_{2}\right) & 0 & 0 \\
0 & 0 & -\sin \left(2 \pi \iota_{1} \omega_{1} / \omega_{2}\right) & 2 \sin ^{2}\left(\pi \iota_{1} \omega_{1} / \omega_{2}\right) & 0 & 0 \\
0 & 0 & 0 & 0 & 2 \sin ^{2}\left(\pi \iota_{1}\right) & \sin \left(2 \pi \iota_{1}\right) \\
0 & 0 & 0 & 0 & -\sin \left(2 \pi \iota_{1}\right) & 2 \sin ^{2}\left(\pi \iota_{1}\right)
\end{array}\right) \\
|\hbar| \\
\left|\begin{array}{cccc}
1-e^{-2 \pi \Omega \iota_{1} / \omega_{2}} & 0 & 0 & 0 \\
0 & 1-e^{2 \pi \Omega \iota_{1} / \omega_{2}} & 0 & 0 \\
0 & 0 & 2 \sin ^{2}\left(\pi \iota_{1} \omega_{1} / \omega_{2}\right) & \sin \left(2 \pi \iota_{1} \omega_{1} / \omega_{2}\right) \\
0 & 0 & -\sin _{2}\left(2 \pi \iota_{1} \omega_{1} / \omega_{2}\right) & 2 \sin ^{2}\left(\pi \iota_{1} \omega_{1} / \omega_{2}\right)
\end{array}\right|
\end{gathered}
$$

hence,

$$
|\hbar|=-16 \sinh ^{2}\left(\pi \Omega \iota_{1} / \omega_{2}\right) \sin ^{2}\left(\pi \iota_{1} \omega_{1} / \omega_{2}\right) \neq 0,
$$

because the ratio of the frequencies is non-resonant with $\pi$. Ti summarize, all of the stated assumptions in Theorem A1 are satisfied by the System (17).

In the proposed system, the map $\xi: \mathbb{R}^{6} \longrightarrow \mathbb{R}^{2}$ can be written as

$$
\xi\left(X_{1}, X_{2}, Y_{1}, Y_{2}, Z_{1}, Z_{2}\right)=\left(Z_{1}, Z_{2}\right),
$$

by evaluating the function

$$
\mathcal{H}\left(\mathcal{Z}_{1}^{0}, \mathcal{Z}_{2}^{0}\right)=\mathcal{H}(\alpha)=\xi\left(\frac{1}{p T_{2}} \int_{0}^{p T_{2}} M_{\mathbf{z}_{\alpha}}^{-1}(t) H_{1}\left(t, \mathbf{x}\left(t, \mathbf{z}_{\alpha}, 0\right)\right) d t\right),
$$

we get $\mathcal{H}\left(\mathcal{Z}^{0}\right)=\left(\mathcal{H}_{1}\left(\mathcal{Z}^{0}\right), \mathcal{H}_{2}\left(\mathcal{Z}^{0}\right)\right)$, where the functions $\mathcal{H}_{k}$ for $k=1,2$ are the ones given in (14). Then, by Theorem A1, we have that, for every simple zero $\mathcal{Z}^{0 *} \in V$ of the system of non-linear functions $\mathcal{H}\left(Z^{0}\right)=0$, we have a periodic solution

$$
\left(X_{1}, X_{2}, Y_{1}, Y_{2}, Z_{1}, Z_{2}\right)(t, \varepsilon)
$$

of System (17), such that

$$
\left(X_{1}, X_{2}, Y_{1}, Y_{2}, Z_{1}, Z_{2}\right)(0, \varepsilon) \longrightarrow\left(0,0,0,0, Z_{1}^{0 *}, Z_{2}^{0 *}\right) \text { when } \varepsilon \longrightarrow 0
$$

Let us changes the variables in System (16), then, one obtains a periodic solution

$$
\left(x_{1}, x_{2}, y_{1}, y_{2}, z_{1}, z_{2}\right)(t, \varepsilon)
$$


of System (17), where

$$
\left(\begin{array}{c}
x_{1}(t, \varepsilon) \\
x_{2}(t, \varepsilon) \\
y_{1}(t, \varepsilon) \\
y_{2}(t, \varepsilon) \\
z_{1}(t, \varepsilon) \\
z_{2}(t, \varepsilon)
\end{array}\right) \rightarrow\left(\begin{array}{c}
0 \\
0 \\
0 \\
0 \\
0.5\left(Z_{1}^{0 *} \cos \left(\omega_{2} t\right)+Z_{2}^{0 *} \sin \left(\omega_{2} t\right)\right) \\
0.86\left(Z_{2}^{0} \cos \left(\omega_{2} t\right)-Z_{1}^{0} \sin \left(\omega_{2} t\right)\right)
\end{array}\right) \text { when } \varepsilon \longrightarrow 0
$$

Thus, periodic solution of System (17) $\left(x_{1}(t, \varepsilon), y_{1}(t, \varepsilon)\right.$, and $z_{1}(t, \varepsilon)$ can be written as

$$
\left(x_{1}, y_{1}, z_{1}\right)(t, \varepsilon) \rightarrow\left(\begin{array}{c}
0 \\
0 \\
0.5\left(Z_{1}^{0 *} \cos \left(\omega_{2} t\right)+Z_{2}^{0 *} \sin \left(\omega_{2} t\right)\right)
\end{array}\right) \text { when } \varepsilon \longrightarrow 0
$$

The previous steps give the complete proof of Theorem 1 .

Proof of Theorem 2. To prove this theorem, we will follow the same steps of proving Theorem 1 . Thus, the periodic solution can be written in the following form:

$$
\left(\begin{array}{c}
0.12\left(Y_{2}^{0} \cos \left(\omega_{1} t\right)-Y_{1}^{0} \sin \left(\omega_{1} t\right)\right) \\
-0.26\left(Y_{1}^{0} \cos \left(\omega_{1} t\right)+Y_{2}^{0} \sin \left(\omega_{1} t\right)\right) \\
-0.41\left(Y_{1}^{0} \cos \left(\omega_{1} t\right)+Y_{2}^{0} \sin \left(\omega_{1} t\right)\right) \\
-0.85\left(Y_{2}^{0} \cos \left(\omega_{1} t\right)-Y_{1}^{0} \sin \left(\omega_{1} t\right)\right) \\
0 \\
0
\end{array}\right) \text { when } \varepsilon \longrightarrow 0 .
$$

Hence, we get a periodic solution $\left(x_{1}, y_{1}, z_{1}\right)(t, \varepsilon)$ of System (13), such that

$$
\left(x_{1}, y_{1}, z_{1}\right)(t, \varepsilon) \rightarrow\left(\begin{array}{c}
0.12\left(Y_{2}^{0} \cos \left(\omega_{1} t\right)-Y_{1}^{0} \sin \left(\omega_{1} t\right)\right) \\
-0.41\left(\Upsilon_{1}^{0} \cos \left(\omega_{1} t\right)+Y_{2}^{0} \sin \left(\omega_{1} t\right)\right) \\
0
\end{array}\right) \text { when } \varepsilon \longrightarrow 0 .
$$

\section{Proof of the Corollaries 1 and 2}

Proof of Corollary 1. Under the aforementioned assumptions in Corollary 1, the nonlinear System (14) can be written as

$$
\begin{aligned}
\mathcal{H}_{1}\left(Z_{1}^{0}, Z_{2}^{0}\right)= & -0.005468749997\left(Z_{1}^{0}\right)^{4} Z_{2}^{0}-0.05177199997\left(Z_{2}^{0}\right)^{2}-0.01093750000\left(Z_{1}^{0}\right)^{2}\left(Z_{2}^{0}\right)^{3} \\
& -0.1553160000\left(Z_{1}^{0}\right)^{2}-0.005468749997\left(Z_{2}^{0}\right)^{5}
\end{aligned}
$$




$$
\begin{aligned}
\mathcal{H}_{2}\left(Z_{1}^{0}, Z_{2}^{0}\right)= & 3.183098861 \times 10^{-12} Z_{1}^{0}\left(6872233931.0\left(Z_{1}^{0}\right)^{2}\left(Z_{2}^{0}\right)^{2}+3436116965.0\left(Z_{1}^{0}\right)^{4}\right. \\
& +3436116965.0\left(Z_{2}^{0}\right)^{4}-65058613960.0 Z_{2}^{0}
\end{aligned}
$$

Then, the solution of the above system is

$$
Z^{0 *}=(0,-2.11) .
$$

Since

$$
\left|\frac{\partial \mathcal{H}}{\partial Z^{0}}\right|=0.21 \neq 0 \text {, when } Z^{0 *}=(0,-2.11)
$$

this solution is simple. Finally, by Theorem 1., we only have one periodic solution for this system and the proof is over.

Proof of Corollary 2. Again, under the aforementioned assumptions in Corollary 2, the non-linear system $\overline{\mathcal{H}}\left(Y^{0}\right)=0$ can be written as

$$
\begin{gathered}
\mathcal{H}_{3}\left(Y_{1}^{0}, Y_{2}^{0}\right)=-0.02699999999 Y_{2}^{0}+0.004649999999 Y_{1}^{0} \\
+0.0009962999998\left(Y_{1}^{0}\right)^{3}+0.0009962999998\left(Y_{2}^{0}\right)^{2} Y_{1}^{0}, \\
\mathcal{H}_{4}\left(Y_{1}^{0}, Y_{2}^{0}\right)=-0.95499999997+0.004649999999 Y_{2}^{0}+0.0009962999998\left(Y_{1}^{0}\right)^{2} Y_{2}^{0} \\
+0.0009962999998\left(Y_{2}^{0}\right)^{3}+0.02699999999 Y_{1}^{0},
\end{gathered}
$$

The above system satisfies the following solution:

$$
Y^{0 *}=(2.590,9.210),
$$

Moreover, since

$$
\left|\frac{\partial \overline{\mathcal{H}}}{\partial Y^{0}}\right|=0.020 \neq 0, \text { when } Y^{0 *}=(2.590,9.210)
$$

the obtained solution is simple, using Theorem 2, one can obtain only one periodic solution for this system, which gives a complete proof.

\section{Conclusions}

The averaging theory is one of the most important perturbation methods that can be used to study the existence and stability of periodic solutions for the ordinary differential equation systems. It is a powerful tool and has proven its effectiveness many times in the literature by examining the existence and stability of the periodicity of dynamical systems, in both physical and engineering sciences.

In this work, the dynamical system of the perturbed spatial Hill problem by quantum corrections, which is called the spatial quantized Hill problem, was analyzed to find the possible periodic solutions. The importance of this problem was stated in the introduction. The differences and similarities among some perturbation approaches, such as the classical perturbation theory, Poincaré-Lindstedt technique, the multiple-scales method, the KB averaging method, and the averaging theory were investigated in the second section. Then, the equilibrium points of the linear system were evaluated. The necessary conditions were analyzed to calculate the periodic solutions emerging from the equilibrium points of the SQHP by using the averaging theory. The application of this theory on the quantized Hill problem has provided interesting and important results about the periodic solution through the proof of Theorems 1 and 2 and their associated corollaries. 
We applied a known theory on a new model to state the new results on such a model. The difficulty of our proofs involved showing all hypotheses of the averaging theory of the dynamical systems for the perturbed spatial quantized Hill problem, in order to apply the theorems of this theory. Changes of variables, obtaining the normal form of this theory, and many technical tricks were needed in this aim, which allowed us to state dynamical information on the perturbed spatial quantized Hill model. Furthermore, this model can be used to develop a lunar theory and the families of periodic orbits in the framework for the spatial quantized Hill problem. Thereby, these applications serve to reinforce the results obtained about these periodic solutions.

Author Contributions: Formal analysis, E.I.A., Z.D. and S.A.; investigation, E.I.A., Z.D., J.L.G.G. and M.H.S.; methodology, E.I.A., Z.D., S.A., J.L.G.G. and M.H.S.; project administration, E.I.A.; software, E.I.A.; Validation, E.I.A., Z.D.; S.A., J.L.G.G. and M.H.S.; visualization, E.I.A.; Writing-original draft, E.I.A.; writing-review and editing, E.I.A., Z.D., S.A., J.L.G.G. and M.H.S. All authors have read and agreed to the published version of the manuscript.

Funding: This work was funded by the National Research Institute of Astronomy and GeophysicsNRIAG, 11421-Helwan, Cairo, Egypt. The first author, therefore, acknowledges his gratitude for NRIAG's technical and financial support. Moreover, this paper was supported by the National Natural Science Foundation of China (NSFC), grant no. 12172322. This paper was partially supported by Ministerio de Ciencia, Innovación y Universidades, grant number PGC2018-097198-B-I00, and by Fundación Séneca of Región de Murcia, grant number 20783/PI/18.

Institutional Review Board Statement: Not applicable.

Informed Consent Statement: Not applicable.

Data Availability Statement: The study does not report any data.

Conflicts of Interest: The authors declare no conflict of interest.

\section{References}

1. Abouelmagd, E.I.; Guirao, J.L.G.; Llibre, J. Periodic orbits for the perturbed planar circular restricted 3-body problem. Discret. Contin. Dyn. Syst. B 2019, 24, 1007. [CrossRef]

2. Hallan, P.; Rana, N. The existence and stability of equilibrium points in the Robe's restricted three-body problem. Celest. Mech. Dyn. Astron. 2001, 79, 145-155. [CrossRef]

3. Abouelmagd, E.I.; Ansari, A.A.; Shehata, M. On Robe's restricted problem with a modified Newtonian potential. Int. J. Geom. Methods Mod. Phys. 2021, 18, 2150005. [CrossRef]

4. Szebehely, V. Theory of Orbit: The Restricted Problem of Three Bodies; Elsevier: Amsterdam, The Netherlands, 2012.

5. Abouelmagd, E.I.; Kalantonis, V.S.; Perdiou, A.E. A Quantized Hill's Dynamical System. Adv. Astron. 2021, 2021. [CrossRef]

6. Abouelmagd, E.I.; Llibre, J.; Guirao, J.L.G. Periodic orbits of the planar anisotropic Kepler problem. Int. J. Bifurc. Chaos 2017, 27, 1750039. [CrossRef]

7. Abouelmagd, E.I. Periodic solution of the two-body problem by KB averaging method within frame of the modified newtonian potential. J. Astronaut. Sci. 2018, 65, 291-306. [CrossRef]

8. Singh, J.; Perdiou, A.; Gyegwe, J.M.; Perdios, E. Periodic solutions around the collinear equilibrium points in the perturbed restricted three-body problem with triaxial and radiating primaries for binary HD 191408, Kruger 60 and HD 155876 systems. Appl. Math. Comput. 2018, 325, 358-374. [CrossRef]

9. Pathak, N.; Thomas, V.; Abouelmagd, E.I. The perturbed photogravitational restricted three-body problem: Analysis of resonant periodic orbits. Discret. Contin. Dyn. Syst. S 2019, 12, 849. [CrossRef]

10. Meyer, K.R. Periodic Solutions of the N-Body Problem; Springer: Berlin/Heidelberg, Germany, 1999; Volume 1719.

11. Sbano, L.; Southall, J. Periodic solutions of the N-body problem with Lennard-Jones-type potentials. Dyn. Syst. 2010, 25, 53-73. [CrossRef]

12. Fusco, G.; Gronchi, G.F.; Negrini, P. Platonic polyhedra, topological constraints and periodic solutions of the classical N-body problem. Invent. Math. 2011, 185, 283-332. [CrossRef]

13. Llibre, J.; Yuan, P. Periodic orbits of the planar anisotropic Manev problem and of the perturbed hydrogen atom problem. Qual. Theory Dyn. Syst. 2019, 18, 969-986. [CrossRef]

14. Llibre, J.; Valls, C. Periodic orbits of the planar anisotropic generalized Kepler problem. J. Math. Phys. 2019, 60, 042901. [CrossRef]

15. Gao, F.; Llibre, J. Periodic orbits of the two fixed centers problem with a variational gravitational field. Celest. Mech. Dyn. Astron. 2020, 132, 1-9. [CrossRef] 
16. Gómez, G. Dynamics and Mission Design Near Libration Points. In Vollume II: Fundamentals: The Case of Triangular Libration Points; World Scientific Monograph Series in Mathematics; World Scientific: Singapore, 2001; Volume 3.

17. Cuntz, M. S-type and P-type habitability in stellar binary systems: A comprehensive approach. I. Method and applications. Astrophys. J. 2013, 780, 14. [CrossRef]

18. Salazar, F.; McInnes, C.; Winter, O. Periodic orbits for space-based reflectors in the circular restricted three-body problem. Celest. Mech. Dyn. Astron. 2017, 128, 95-113. [CrossRef]

19. Murakami, S. Almost periodic solutions of a system of integrodifferential equations. Tohoku Math. J. 1987, 39, 71-79. [CrossRef]

20. Palmer, K.J. Exponential dichotomies for almost periodic equations. Proc. Am. Math. Soc. 1987, 101, 293-298. [CrossRef]

21. Cooke, K.L.; Wiener, J. A survey of differential equations with piecewise continuous arguments. In Delay Differential Equations and Dynamical Systems; Springer: Berlin/Heidelberg, Germany, 1991; pp. 1-15.

22. Dads, E.A.; Ezzinbi, K. Existence of positive pseudo-almost-periodic solution for some nonlinear infinite delay integral equations arising in epidemic problems. Nonlinear Anal. Theory Methods Appl. 2000, 41, 1-13. [CrossRef]

23. Guirao, J.L.; Llibre, J.; Vera, J.A. On the dynamics of the rigid body with a fixed point: Periodic orbits and integrability. Nonlinear Dyn. 2013, 74, 327-333. [CrossRef]

24. Feddaoui, A.; Llibre, J.; Berhail, C.; Makhlouf, A. Periodic solutions for differential systems in R 3 and R 4. Appl. Math. Nonlinear Sci. 2021, 6, 373-380. [CrossRef]

25. Krylov, N.; Bogolyubov, N. Introduction to Non-Linear Mechanics; Princeton University Press: Princeton, NJ, USA, 1947.

26. Malkin, I.G. Some Problems in the Theory of Nonlinear Oscillations; Technical Information Service; US Atomic Energy Commission: Washington, DC, USA, 1959; Volume 1.

27. Dobrokhotov, S.Y. Resonances in multifrequency averaging theory. In Singular Limits of Dispersive Waves; Springer: Berlin/Heidelberg, Germany, 1994; pp. 203-217.

28. Lehman, B.; Weibel, S.P. Fundamental theorems of averaging for functional differential equations. J. Differ. Equ. 1999, 152, 160-190. [CrossRef]

29. Llibre, J. The averaging theory for computing periodic orbits. In Central Configurations, Periodic Orbits, and Hamiltonian Systems; Springer: Berlin/Heidelberg, Germany, 2015; pp. 1-104.

30. Bogoliubov, N. Asymptotic Methods in the Theory of Non-Linear Oscillations; Gordon \& Breach: New York, NY, USA, 1961.

31. Celletti, A. Stability and Chaos in Celestial Mechanics; Springer: Berlin/Heidelberg, Germany, 2010.

32. Nayfeh, A.H. Introduction to Perturbation Techniques; John Wiley \& Sons: Hoboken, NJ, USA, 2011.

33. Alshaery, A.; Abouelmagd, E.I. Analysis for the spatial quantized three-body problem. Results Phys. 2020, 17, 103067. [CrossRef]

34. Henrard, J.; Navarro, J.F. Spiral structures and chaotic scattering of coorbital satellites. Celest. Mech. Dyn. Astron. 2001, 79, 297-314. [CrossRef]

35. De Bustos, M.; Guirao, J.; Vera, J. The spatial Hill lunar problem: Periodic solutions emerging from equilibria. Dyn. Syst. 2017, 32, 340-353. [CrossRef] 